\title{
REAL-TIME CHARACTER ANIMATION USING PUPPET METAPHOR
}

\author{
Yoshihiro Okada \\ Graduate School of Information Science and Electrical Engineering, Kyushu University \\ 6-1 Kasuga-koen, Kasuga, Fukuoka, 816-8580 Japan \\ e-mail: okada@i.kyushu-u.ac.jp, Phone: +81-92-583-7632, Fax: +81-92-583-7632
}

\begin{abstract}
For computer animation creation, character design is a very important factor but very hard work. Especially its motion design is very laborious work. So the authors propose new motion design method using a puppet metaphor and this paper introduces its prospective application examples. The puppet show is one of very popular entertainments. Puppets are not real characters like human actors/actresses and they cannot take real actions. However the puppet show has enough entertainment aspects. Moreover, the puppet is also children toy and it is easy to manipulate for even children. Then the authors employ the puppet metaphor for motion design of computer animation creation.
\end{abstract}

Key words: Character animation, Puppet show, Virtual puppet, Virtual Reality, Interface

\section{INTRODUCTION}

Advances in recent computer hardware technology have made possible $3 \mathrm{D}$ rendering images in real-time. However, it is still difficult for end-users to develop 3D graphics software. For this reason, Okada and Tanaka developed a 3D prototype system called IntelligentBox [1]. IntelligentBox is a component based construction system. Its application fields include various kinds, e.g., 3D game construction [2, 3], collaborative virtual environment construction [4] and so on. IntelligentBox also has aspects as an interactive animation system $[1,5]$.

For computer animation creation, character design is a very important factor but very hard work. Especially its motion design is very laborious work. So this paper proposes new motion design method using a puppet metaphor and introduces its prospective application examples, e.g., CG puppet show theatre, 3D fighting game etc. The puppet show is one of very

The original version of this chapter was revised: The copyright line was incorrect. This has been corrected. The Erratum to this chapter is available at DOI: 10.1007/978-0-387-35660-0_65 
popular entertainments. Puppets are not real characters like actual human actors/actresses and they cannot take real actions. However the puppet show has enough entertainment aspects. Moreover, the puppet is also children toy and it is easy to manipulate for even children. For this reason, we employ the puppet metaphor for motion design of computer animation creation. We have developed fundamental mechanisms for such a virtual puppet using IntelligentBox. As mentioned above, IntelligentBox is a component based construction system that provides various functional components called boxes. The proposed virtual puppet is also developed as a composite box. IntelligentBox also provides a particular component called RoomBox [4]. Using RoomBox, our virtual puppet will become available through Internet. Strictly speaking, multiple users using a different computer can play his/her CG represented puppet collaboratively. We use a data-glove and one magnetic-based motion sensor as controlling devices for the puppet. Then the user controls his/her puppet dynamically and interactively. Its application fields include interactive game development beside computer animation creation as described in section 4 .

There are many researches on motion generation for computer animation. Witkin and Kass proposed concept of spacetime constraints [6]. After that, many research papers based on spacetime constraints were published [7]. IK (Inverse Kinematics) is one of the other popular methods for efficient motion generation. The motion path functionality is also a popular technique to intuitively define movement of a character's center of mass. Furthermore, the use of motion capture systems has become common to generate motion data for computer animation creation. Noser and Thalmann proposed virtual tennis game environment using a full-body motion capture system as a realtime motion input interface [8]. In this paper, we propose a real-time motion design method for character animation using only one motion sensor and a data-glove. We have never met such approaches.

The remainder of this paper is organized as follows. Section 2 explains essential mechanisms of IntelligentBox. Section 3 describes fundamental mechanisms of the puppet. Section 4 shows several prospective application examples. Finally Section 5 concludes this paper.

\section{OVERVIEW OF INTELLIGENTBOX}

As introduction of IntelligentBox, this section explains its essential mechanisms briefly. 


\subsection{Model-Display Object (MD) Structure}

As shown in Figure 1, each box consists of two objects, a model and a display object. This structure is called an MD (Model-Display object) structure. A model holds state values of a box. They are stored in variables called slots. A display object defines how the box appears on a computer screen and defines how the box reacts to user operations.

Figure 1 also shows messages between a display object and a model. This is an example of a RotationBox. A RotationBox has a slot named 'ratio' that holds a rotation angle value. Through direct manipulations on a box, its associated slot value changes. Furthermore, its visual image simultaneously changes according to the slot value change. In this way a box reacts to a user's manipulations according to its functionality.

\subsection{Message-sending Protocol for Slot Connections}

Figure 2 illustrates a data linkage concept among boxes. Each box has multiple slots. Its one slot can be connected to one of the slots of other box. This connection is called a slot connection. The slot connection is carried out by three messages when a parent-child relationship exists between two boxes. They are a set message, a gimme message and an update message. These messages have the following formats:

(1) Parent box set $<$ slotname $><$ value $>$.

(2) Parent box gimme <slotname $>$.

(3) Child box update.

A $<$ value $>$ in a format (1) represents any value, and a $<$ slotname $>$ in formats (1) and (2) represents a user-selected slot of the parent box that receives these two messages.

A set message writes a child box slot value into its parent box slot. A gimme message reads a parent box slot value and sets it into its child box slot. Update messages are issued from a parent box to all of its child boxes to tell

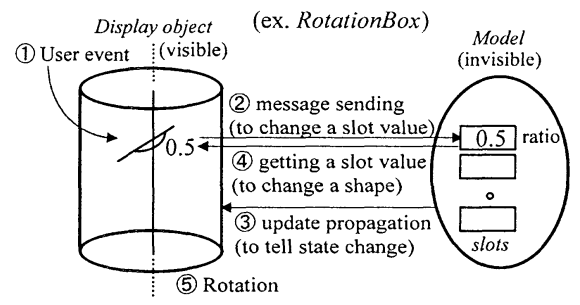

Figure 1. An $M D$ structure of a box and its internal messages

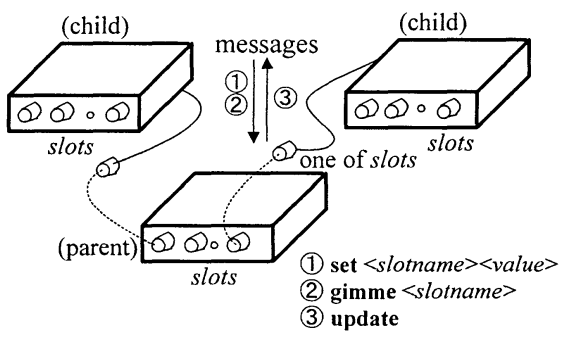

Figure 2. Standard messages between boxes 
them that the parent box slot value has changed. In this way, these three messages connect a child box slot and its parent box slot, and combine their two functionalities.

\section{REAL-TIME PUPPET CONTROL}

Our proposed puppet is controlled using a data-glove and one magneticbased motion sensor device. Then only one-hand motion manipulates the puppet.

\subsection{Component Structure of Puppet Model}

Figure 3 (left) shows components of a typical puppet model. This model consists of 17 joints. Each joint is a 3DRotationBox. 3DRotationBox has three DOF (Degrees Of Freedom) and then it rotates along x,y,z-axes. IntelligentBox provides a particular box called SGJBox. This box receives data sent from a data-glove device. We use Nissho Electronics Corporation's Super Glove Jr. as shown in Figure 3 (right). This device generates ten joint angles data. Each of these angles is applied to some specific joints of the puppet. Then the real-hand motion controls the puppet motion in real-time. IntelligentBox also provides another particular box called ISOTRACKBox. This box receives data sent from a magnetic based motion sensor, Polhemus Inc. 3SPACE ISOTRACK II. This device generates one six degrees of freedom data. The position and orientation of the puppet change according to this data.

As mentioned above, the puppet model, a human-like model, has 17 joints. However, the data-glove generates only ten joint angles data. To control the puppet motion by only one-hand motion, it needs a certain

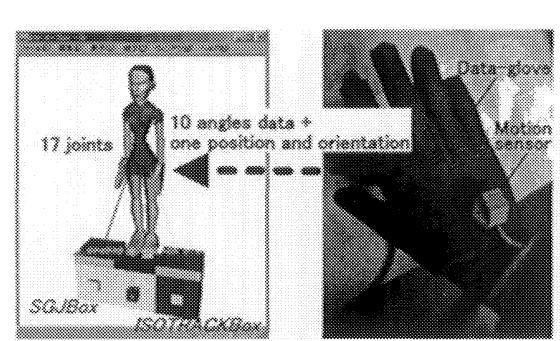

Figure 3. Puppet model and its control interface

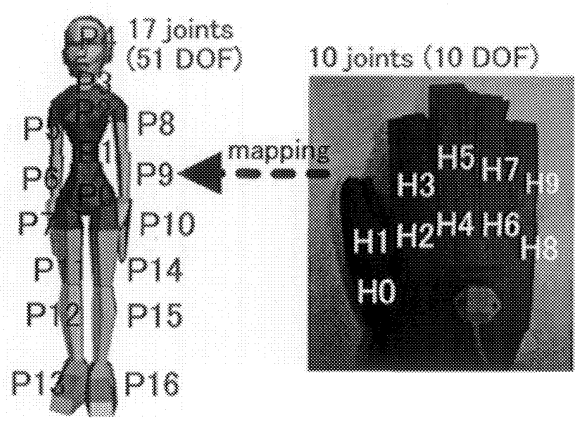

Figure 4. Puppet joints and hand joints 
mapping scheme between 17 joints of the puppet and ten angles data of the data-glove as shown in Figure 4. One of our research purposes is to define optimum mapping between the data-glove data and the puppet model's joints angles for controlling the puppet easily and accurately.

\subsection{Motion Control Example}

Figure 5 shows four poses of the hand and their four corresponding poses of the puppet. We define Pose 1 is an initial pose since a paper shape of the hand seems more natural rather than a stone shape. In this example case, a mapping scheme is specified as shown in Table 1. A Table column 'PJoint' indicates each joint of the puppet, and 'HJoint' indicates each joint of the hand. For example, Joint P11 and P12, a right upper leg and lower leg of the puppet, are controlled by a little finger of the hand. Strictly speaking, each joint of the puppet has $\mathrm{x}, \mathrm{y}, \mathrm{z}$-angle values so that only $\mathrm{x}$-angle of P11 is controlled by $\mathrm{H} 8$ angle value. X-angle of $\mathrm{P} 12$ is also controlled by $\mathrm{H} 9$ angle value. However, its direction is opposite to P11 since its 'Dir' column is '-'. Actually the following substitutions (1) and (2) are done.

$$
P 11_{x}=H 8 \text { (1) } \quad P 12_{x}=-H 9
$$

Here, $P 11_{x}$ means $\mathrm{x}$-angle of joint $\mathrm{P} 11$, and $P 12_{x}$ means $\mathrm{x}$-angle of joint $\mathrm{P} 12$.
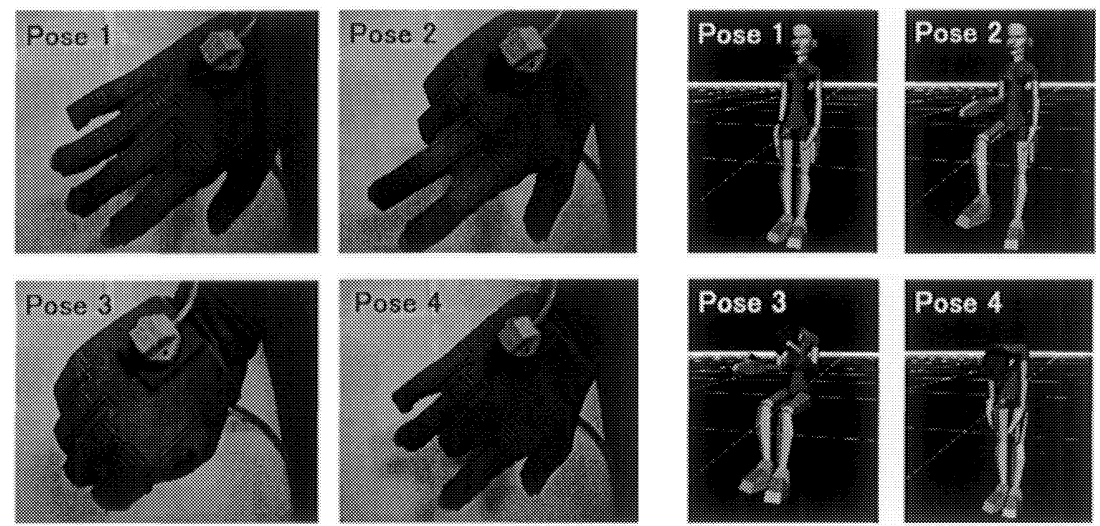

Figure 5. Four one-hand poses and their corresponding puppet poses

Table 1. Mapping table example

\begin{tabular}{|l|l|l|l|l|l|l|}
\hline PJoint & Dir. & HJoint & & PJoint & Dir. & HJoint \\
\hline P0 & & & & P9 & + & H3 \\
\hline P1 & & & & P10 & & \\
\hline P2 & + & H4 & & P11 & + & H8 \\
\hline P3 & + & H5 & & P12 & - & H9 \\
\hline
\end{tabular}




\begin{tabular}{|l|l|l|l|l|l|l|}
\hline P4 & & & & P13 & & \\
\hline P5 & + & H6 & & P14 & + & H0 \\
\hline P6 & + & H7 & & P15 & - & H1 \\
\hline P7 & & & & P16 & & \\
\hline P8 & + & H2 & & & & \\
\hline
\end{tabular}

In this way, this system becomes applicable to various applications by providing multiple mapping tables each of which is suitable for each different application.

\subsection{Puppets except Human-like Model}

IntelligentBox also provides an FFD (Free Form Deformation) function for soft object animation as a particular box called FFDControlBox. For example, Figure 6 shows Triceratops animation using FFDContorlBox. Its detail is written in the paper [1]. Using this box, the user can employ any arbitral shaped model as his/her puppet.
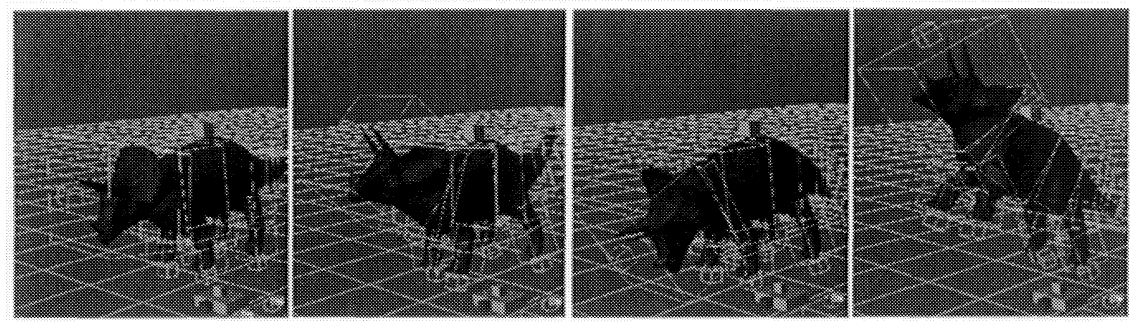

Figure 6. Triceratops animation using FFDControlBox

\section{APPLICATION EXAMPLES}

This section briefly introduces several prospective application examples especially included in the entertainment field.

\subsection{CG Puppet Show Theatre}

As described previously, IntelligentBox has provided a network communication facility as a particular box called RoomBox [4]. As shown in Figure 7, RoomBox manages user operation events and virtually provides a shared 3D space in which several users can work collaboratively. The composite component shown in the figure 3 is also available in such a shared $3 \mathrm{D}$ space. Then several users using a different computer can control his/her 
puppet in the shared 3D space simultaneously and play collaboratively. If they play a given role of an actor/actress, this means the CG puppet show.

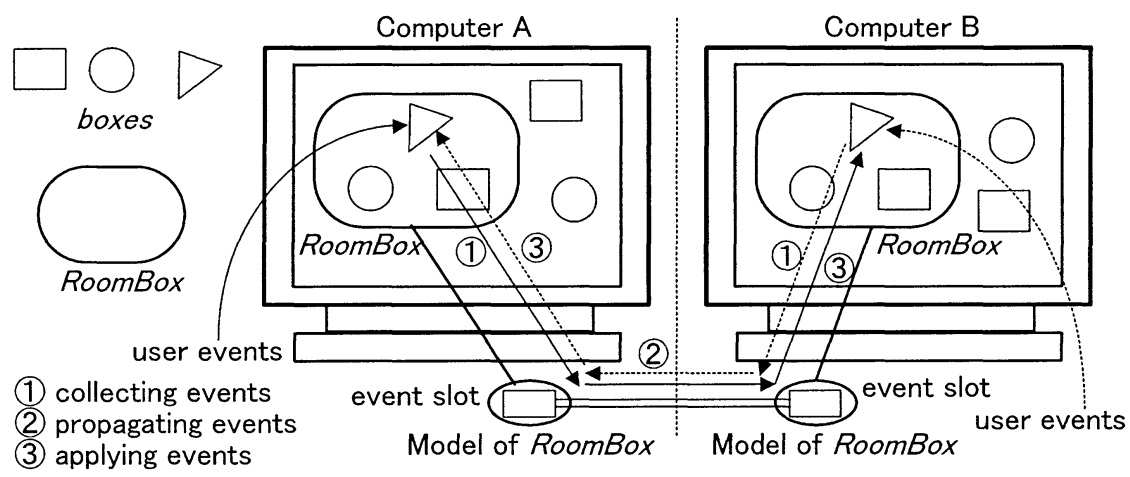

Figure 7. Message flow between two RoomBoxes for network collaboration

\subsection{Interface for 3D Video Games}

Sega VirtuaFighter ${ }^{\mathrm{TM}}$ is a very famous video game as a 3D fighting game. To specify fighting actions and to prepare a mapping table for them will make possible to develop a 3D fighting game as shown in Figure 8. This prospective game will accept the user's hand gesture as input data for controlling his/her fighter instead of a joystick.

Sega SpaceChannel5 ${ }^{\mathrm{TM}}$ is another video game, a musical action adventure game. The main aim of this game is to control a player's doll to take the same action/motion as enemy's action/motion like a dance. This prospective game will accept the user's hand gesture as input data for controlling his/her doll instead of a joystick.

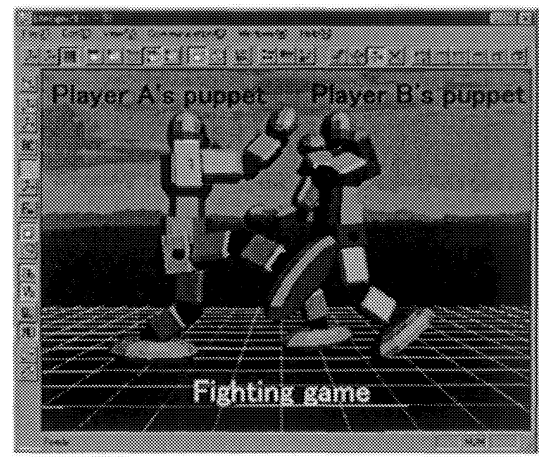

Figure 8. Image of 3D fighting game

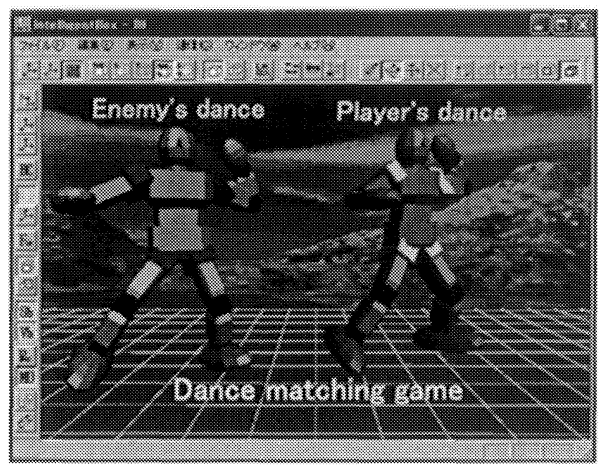

Figure 9. 3D game like SpaceChannel5 ${ }^{\mathrm{TM}}$ 


\section{CONCLUDING REMARKS}

This paper proposed new motion design method using a puppet metaphor and introduced its prospective application examples especially included in the entertainment field. Actually we have to develop such application examples to clarify usefulness of a puppet metaphor. However we have not yet done it since we have just started this research. Currently we are developing such application examples and we will evaluate our proposed puppets. We will soon report its results and new findings.

\section{ACKNOWLEDGEMENT}

This work is partially supported by research grant of Ministry of Education, Culture, Sports, Science and Technology of Japan.

\section{REFERENCES}

[1] Okada, Y. and Tanaka, Y., IntelligentBox: A Constructive Visual Software Development System for Interactive 3D Graphic Applications, Proc. of Computer Animation '95, IEEE Computer Society Press, pp. 114-125, 1995.

[2] Okada, Y., Itoh, E. and Hirokawa, S., IntelligentBox: Its Aspects as a Rapid Construction System for Interactive 3D Games, Proc. of First International Conference on Intelligent Games and Simulation, SCS Publication, pp. 22-26, 2000.

[3] Okada, Y., Component Based Motion Editing Environment for Game Character Design, Proc. of Second International Conference on Intelligent Games and Simulation, SCS Publication, pp. 22-26, 2001.

[4] Okada, Y. and Tanaka, Y., Collaborative Environments in IntelligentBox for Distributed 3D Graphics Applications, The Visual Computer (CGS special issue), Vol. 14, No. 4, pp. 140-152, 1998.

[5] Okada, Y. and Tanaka, Y., IntelligentBox: Its Aspect as an Interactive Animation System, Proc. of SCI'99/ISAS'99, Vol.2, pp. 198-201, 1999.

[6] Witkin, A. and Kass, K., 1988 : Spacetime constraints, Proc. of SIGGRAPH'88, pp. 159-168, 1988.

[7] Gleicher, M., Motion editing with spacetime constraints, Proc. of SIGGRAPH'97, pp. 139-148, 1997.

[8] Noser, H. and Thalmann, D., Sensor Based Synthetic Actors in a Tennis Game Simulation, Proc. of Computer Graphics International '97, IEEE Computer Society Press, pp.189-198, 1997. 